\title{
Controlled Synthesis of Au Nanoparticles by Modified Polyol Methods: Determination of Their Size, Shape, and Crystal Structure
}

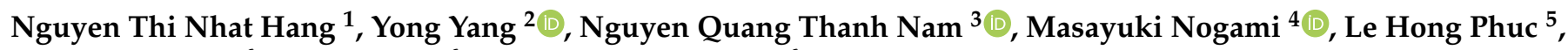 \\ Nguyen Huu Tri ${ }^{6}$, Ho Van Cuu ${ }^{6}$ and Nguyen Viet Long ${ }^{6, *}$
}

1 Institute of Applied Technology, Thu Dau Mot University, Thu Dau Mot City, Vietnam; hangntn@tdmu.edu.vn

2 State Key Laboratory of High Performance Ceramics and Superfine Microstructures, Shanghai Institute of Ceramics, Chinese Academy of Sciences, 1295 Dingxi Road, Shanghai 200050, China; yangyong@mail.sic.ac.cn

3 Faculty of Chemical Engineering, Ho Chi Minh City University of Technology, 268 Ly Thuong Kiet, District 10, Ho Chi Minh City 700000, Vietnam; thanhnam272000@gmail.com

4 Institute of Research and Development, Duy Tan University, Da Nang 55000, Vietnam; mnogami@mtj.biglobe.ne.jp

5 Ho Chi Minh City Institute of Physics, 1 Mac Dinh Chi Str., Ben Nghe, District 1, Ho Chi Minh City 700000, Vietnam; lhphuc@hcmip.vast.vn

6 Faculty of Electronics and Telecommunications, Saigon University, 273 An Duong Vuong, District 5, Ho Chi Minh City 700000, Vietnam; huutri_sgu@yahoo.com (N.H.T.); cuu_ho_van@yahoo.com (H.V.C.)

Citation: Hang, N.T.N.; Yang, Y.; Nam, N.Q.T.; Nogami, M.; Phuc, L.H.; Tri, N.H.; Cuu, H.V.; Long, N.V. Controlled Synthesis of $\mathrm{Au}$ Nanoparticles by Modified Polyol Methods: Determination of Their Size, Shape, and Crystal Structure. Crystals 2021, 11, 1297. https:// doi.org/10.3390/cryst11111297

Academic Editors: Adele Moatti, Jeffery A. Aguiar, Ritesh Sachan, Rajratan Basu and Borislav Angelov

Received: 2 September 2021

Accepted: 21 October 2021

Published: 26 October 2021

Publisher's Note: MDPI stays neutral with regard to jurisdictional claims in published maps and institutional affiliations.

Copyright: (c) 2021 by the authors Licensee MDPI, Basel, Switzerland. This article is an open access article distributed under the terms and conditions of the Creative Commons Attribution (CC BY) license (https:// creativecommons.org/licenses/by/ $4.0 /)$.
* Correspondence: nguyenvietlong@sgu.edu.com; Tel.: +84-(0)946293304

\begin{abstract}
We successfully prepared Au nanoparticles using the modified polyol methods and design of experiments. The desirable crystal structure and particle size of Au nanoparticles with various kinds of polyhedral and spherical shapes as well as various kinds of their morphologies or complete and rough spherical crystal surfaces were experimentally predicted in TEM and XRD measurements according to the theoretical calculation and data. The fine crystal formation of Au nanostructures by modified polyol methods with EG, PVP, and $\mathrm{NaBH}_{4}$ is of great importance to their practical applications. Our research shows that the critical nucleation, growth, and formation of sizes, shapes, and morphologies of Au nanoparticles were experimentally discussed in modified polyol methods and design of experiments. In this context, our particle size calculation can hold the greatest attraction for researchers in the field of nanoparticles.
\end{abstract}

Keywords: gold nanoparticles; modified polyol methods; crystal; surface plasmon resonance; biomedical applications; assembly; collision

\section{Introduction}

So far, metal nanoparticles have been widely studied because of their practical applications in electronics and telecommunications, catalysis, energy and environment, health and clinic applications [1-7]. The unique optical properties of $\mathrm{Au}, \mathrm{Ag}$, and $\mathrm{Cu}$ single-metal nanoparticles (electron clouds) under suitable light excitation (formation of an electric dipole) are the key foundation of nanoscale electronics and telecommunications [4,5]. In their controlled synthesis, modified polyol methods have been previously discussed in the relationship of Au precursors, reducing agents, protective, and structure-controlling agents in ethylene glycol (EG) or polyEG (PEG) [1-7]. Their promising applications of functional nanoparticles are used in catalysis and nanobiomedicine $[7,8]$. The common synthetic methods of metal nanoparticles suggest that Au or gold nanoparticles can be effectively synthesized by modified polyol methods [1-7]. Recently, Au and Au-based nanoparticles have been potentially used for catalysis, energy, and environment, Au nanoparticles in the diagnosis and treatment of melanoma cancer [9], and fluorescence-based assays in cancer and non-cancerous cells. Therefore, many researchers have proposed methods of controlling the nanosize ranges and the definite shapes of Au nanoparticles by changing 
the experimental conditions [7,8]. In biomedical applications, Au nanoparticles are used as an antigen carrier and an immunological agent in immunization to prepare antibodies in vivo [9]. However, the highest biocompatibility is silver nanoparticles for plants, animals, and humans [9]. In comparison with the traditional sol-gel method for the synthesis of nanomaterials in alcohol, the polyol method is used to synthesize nanomaterials with typical industrial solvents such alcohols, ethylene glycol (EG), and polyethylene glycol (PEG) [2-10]. Both sol-gel and polyol methods can lead to homogeneous nanomaterials and nanoparticles because homogeneous industrial solvents and polymers are under mixing continuously. In the controlled synthesis of nanoparticles, EG and PEG are commonly utilized for the effective synthesis of metal nanomaterials and nanoparticles [11]. Both EG and PEG solvents are effectively used with the addition of a diversity of various reductants to $\mathrm{Au}$ precursors, and the addition of a diversity of various protective polymers as a stabilizer for nanoparticles such as poly(vinylpyrrolidone) (i.e., PVP) or hexadecyltrimethylammonium bromide (i.e., $\mathrm{CTAB}$ ), and tetradodecylammonium bromide (i.e., TCAB) in various solvents such as EG or PEG or various polyols or various kinds of water (i.e., $\mathrm{H}_{2} \mathrm{O}$ ) [12]. The synthetic methods, structures, properties, and applications of Au nanoparticles have been discussed in various works [13-21]. Among the promising applications, the very interesting phenomena and optical properties of surface plasmon resonance (SPR) and surface-enhanced Raman scattering (SER) have been utilized in biomedicine [1-6]. In the present research, we synthesized Au nanoparticles by modified polyol processes with the addition of $\mathrm{NaBH}_{4}$ in EG and PVP solvents according to suitable time and temperature. We used two measurements (i.e., a combination of XRD and TEM methods), in order to characterize the as-prepared Au nanoparticles. The critical issues of the particle size, the particle shape, and the face-centered cubic (FCC) crystal structure of the as-prepared Au nanoparticles are clearly underlined in detailed discussion.

\section{Materials and Methods}

Chemicals for synthesis were poly(vinylpyrrolidone) (PVP), $\mathrm{HAuCl}_{4} \cdot 3 \mathrm{H}_{2} \mathrm{O}, \mathrm{NaBH}_{4}$, and ethylene glycol (EG). All chemicals are available from Aldrich or Sigma-Aldrich and meet the synthetic requirements. The addition of $\mathrm{NaBH}_{4}$ was used for the fast crystal formation of $\mathrm{Au}$ nanoparticles from $\mathrm{HAuCl}_{4}$. EG was the homogeneous solvent that was used as both a solvent and weak reducing agent. Ethanol, acetone, and hexane were appropriately used for cleaning the very thin layers of PVP covering the surfaces of PVP-protected Au nanoparticles [6-10]. Here, all chemicals were used without any further purification. In one typical process, an amount of EG, an amount of $0.375 \mathrm{M}$ PVP, an amount of $0.0625 \mathrm{M} \mathrm{HAuCl}_{4}$, and $\mathrm{NaBH}_{4}$ were added in a three-neck flask or multineck flask with a reflux system [6]. In the synthetic methodology, the addition of $\mathrm{Au}, \mathrm{PVP}, \mathrm{NaBH}_{4}$, and other precursors was carried out until all their amounts were completely used with continuous stirring (Scheme 1). The good reduction of $\mathrm{HAuCl}_{4}$ by both $\mathrm{EG}$ and $\mathrm{NaBH}_{4}$ occurred according to our design processes, which depended on the technique and experience of the experimenter [10-13]. In order to remove PVP that existed on the surfaces of asprepared Au nanoparticles, they were cleaned with an acetone and ethanol/hexane mixture. Finally, clean Au nanoparticles were successfully obtained by the centrifugation procedure. For a successful synthesis of the modified polyol method, the ratios of the volumes and concentrations of the reagents (Au precursors for crystal formation of Au nanoparticles, EG solvent, PVP polymer for the protection of Au nanoparticles, $\mathrm{NaBH}_{4}$, etc.) need to be selected suitably according to the process used. In the study of crystal formation, both XRD and TEM methods were effectively used for the investigation of the crystal structure of as-prepared Au nanoparticles by the polyol process. It is ensured that the final products of $\mathrm{Au}$ nanoparticles were studied by TEM and XRD measurements after the removal of PVP. Here, the X-ray diffraction (XRD) method was used for structural information by identifying the crystalline phases of Au nanoparticles within the prepared samples. Here, the Au nanoparticles with the removal of PVP were set on a glass substrate. We used an X-ray diffractometer that was Rigaku D (max $2550 \mathrm{~V})$ operating at $40 \mathrm{kV} / 200 \mathrm{~mA}$, and 
Cu-K $\alpha 1$ radiation at $1.54056 \AA$. In this study, the FCC crystal structure of Au nanoparticles was proven. In the surface analysis, the JEM-2100F analytical electron microscope was used with standard magnification up to 1,500,000 times, ultra-high image quality and resolution operating at $200 \mathrm{kV}$, around a $0.1 \mathrm{~nm}$ lattice resolution, and around $0.19 \mathrm{~nm}$ point resolution. The images of $\mathrm{Au}$ nanoparticles by JEM-2100F were used to investigate the interesting properties of the size and the shape of Au nanoparticles.

(a)

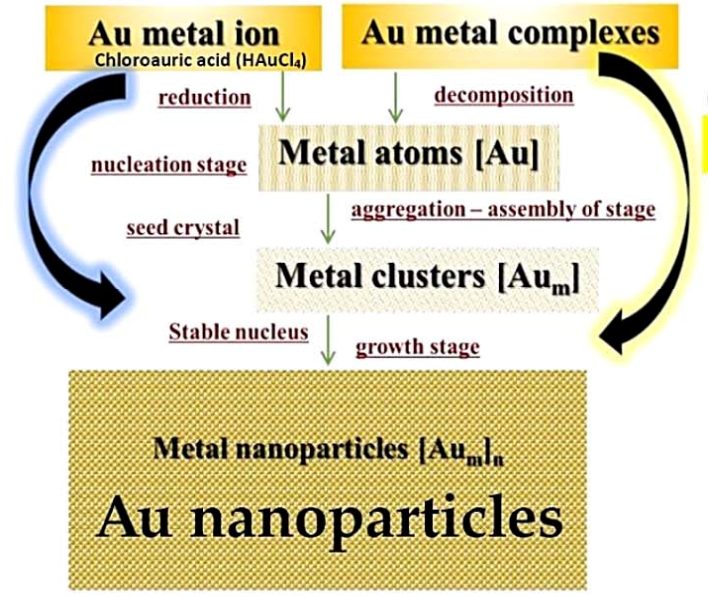

(b)

$0.375 \mathrm{M} \mathrm{HAuCl}_{4}$

PVP

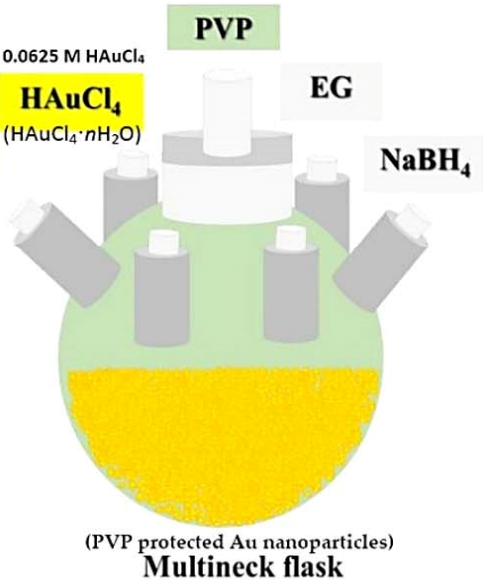

(c)

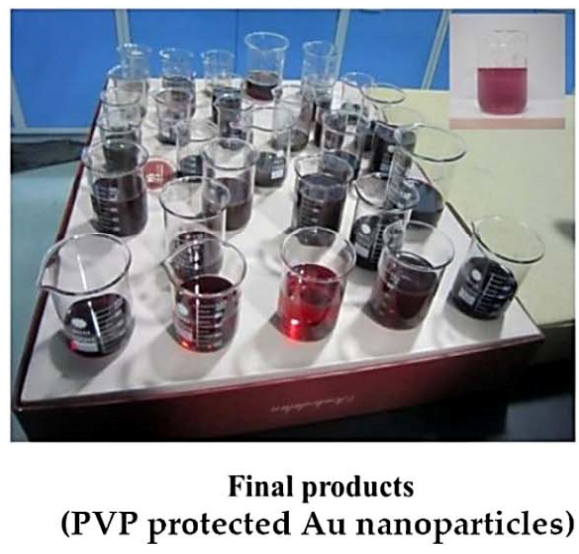

Scheme 1. Experimentally synthetic models and controlled synthesis of as-prepared Au nanoparticles by modified polyol methods. (a) The formation of Au nanoparticles. (b) Multineck flask model. (c) The final products of PVP-Au nanoparticles in EG.

\section{Results and Discussion}

\subsection{Determination of Crystal Structure}

The exact determination of the crystal structure of Au nanoparticles needs to be undertaken in any experiment. The Au crystal structure of a typical XRD pattern was described by the positions, intensities, and shapes of Bragg reflections from $32.5^{\circ}$ to $95^{\circ}$ corresponding to the experiment, and from $0^{\circ}$ to $120^{\circ}$ corresponding to the theory (Figure 1 ). The crystal structure of the as-prepared Au nanoparticles was very good, which was found in the crystal formation in high crystallization in EG solvent in this polyol method. Therefore, XRD was effectively used to determine the crystal structure of the clean Au nanoparticle PVP formed in EG with the removal of PVP. The results have shown the formation of the crystal structure of the as-prepared Au nanoparticles with high crystallization (Figure 1a). It indicates that all the as-synthesized Au nanoparticles have a crystal structure in high crystallization. In analysis and investigation, we showed the crystal structure of one of the most typical samples prepared by modified polyol methods with the use of a controlling agent (i.e., $\mathrm{NaBH}_{4}$ (reducing agents), $\mathrm{AgNO}_{3}$ (i.e., crystal structure-controlling agents), PVP (i.e., protecting agents) $[6,10])$. It is known that the crystal formation of Au nanoparticles was confirmed in various homogeneous polyol liquids (i.e., EG, polyEG ... ). It is certain that Au nanoparticles have a FCC crystal structure from experimental observations and theory [6,13-21]. There is a comparison between one sample by XRD where the diffraction peaks at $2 \theta$ angles of the prepared Au nanoparticles with the fcc structure were similar to other studies [6,14-17]. Obviously, Au nanoparticles were determined by the positions of the important diffraction peaks that were shown with the $2 \theta$ angles at $38.20^{\circ}, 44.40^{\circ}, 64.60^{\circ}$, $77.54^{\circ}$, and $81.72^{\circ}$, corresponding to the fine crystal planes at (111), (200), (220), (311), and (222) or more Miller indices. However, the intensities of their diffraction angles and peaks were very different. In addition, their similar crystal parameters were listed in the PDF-04-0784 standard sample of Au as well as the powder diffraction pattern from the $\mathrm{Au}$ crystal model of ten-unit cells. For the determination of crystal structure, the typical values of the distances between atomic planes of Au nanoparticles were about 2.35, 2.03, 1.44, 1.23, 
and $1.17 \AA$, corresponding to the (111), (200), (220), (311), and (222) planes, respectively. It is important to note that the intensity of the strongest line, as shown in Figure 1, is the so-called (111) plane. In addition, high crystallization of Au nanoparticles coincided with previous works [15-17]. Therefore, the fine crystal phase of as-prepared Au nanoparticles by our polyol process with the addition of $\mathrm{NaBH}_{4}$ was experimentally confirmed because a mixture of EG and PVP was very highly homogeneously mixed in the crystal formation of Au nanoparticles. In Figure 1, the typical crystal parameters such as the values of ( $h \mathrm{k} l$ ), $\mathrm{d}, \theta, 2 \theta$, and I are measured or calculated by nanostructures of Au nanoparticles show very good agreement between the theory and experiment.

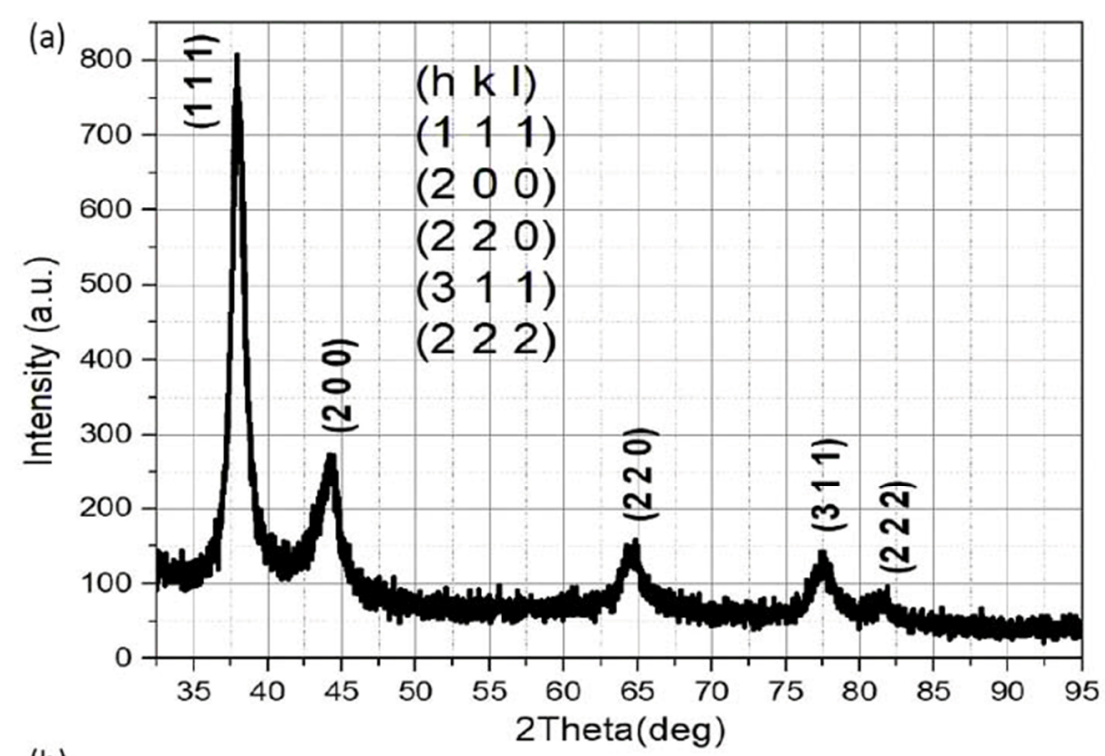

(b)

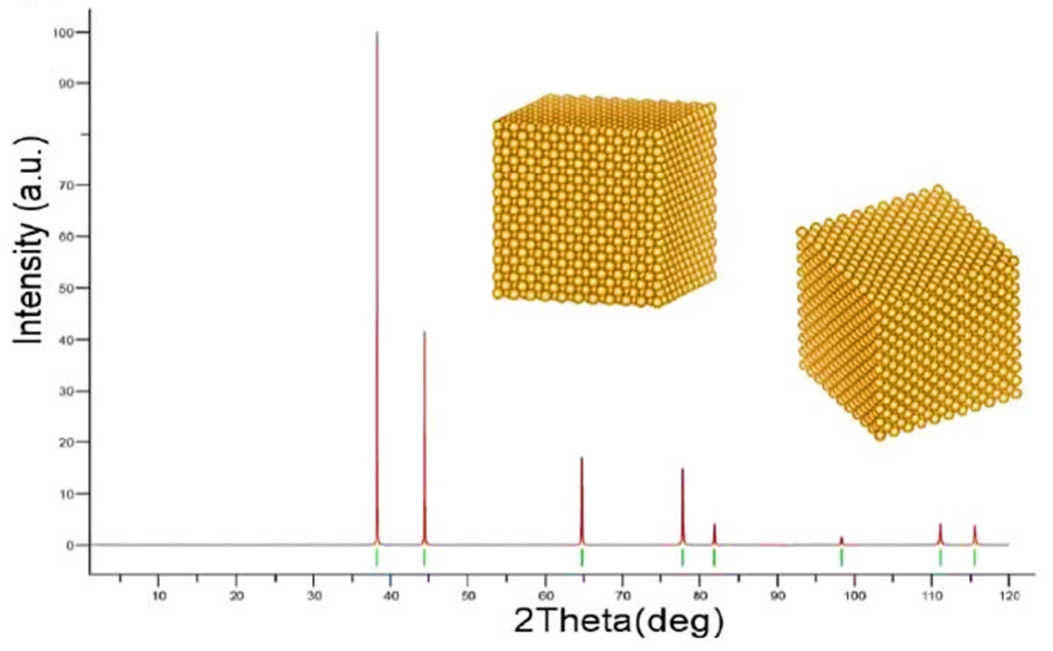

Figure 1. (a,b) Experimental XRD pattern of as-prepared Au nanoparticles, crystal structure, and crystallographic and unit cells by using Jade 6.0 version (Materials Data, Inc., Livermore, CA, USA), and Vesta software (two models of the fcc ten-unit cells of gold atoms) [22-24].

Recently, authors have successfully synthesized Au nanoparticles by seeded growth methods [1-6]. In general, they have used various kinds of capping agents [12] that are different from the use of PVP as a capping agent [1-12]. Recently, $\mathrm{NaBH}_{4}$ has been used in a different way for the controlled synthesis of Au nanoparticles (i.e., fast, moderate, and slow reduction of Au precursor into Au nanoparticles [1-6]). In important considerations, researchers have used the $\mathrm{NaBH}_{4}$ reducing agent for the synthesis of $\mathrm{Au}$ nanoparticles $[2,3,6,12]$. TEM and high resolution (HR) TEM results in typical works of 
Au nanoparticles were compared to our present results. In addition, SPR and SER optical properties of the Au nanoparticles in solvents and solutions are very crucial in promising applications for plasmonic and Raman sensing [4-6]. In size and shape analysis, we only used one of the most typical samples for TEM investigation.

\subsection{Determination of Size and Shape of As-Prepared Au Nanoparticles}

Figure 2 shows the TEM images of as-prepared Au nanoparticles with the various forms of the fascinating shapes and morphologies such as spheres, polyhedrons, plates, rods, and other undefined shapes in the nanosize range of $60 \mathrm{~nm}$. The various kinds of the sizes of Au nanoparticles were calculated in the ranges of $60 \mathrm{~nm}, 50 \mathrm{~nm}, 40 \mathrm{~nm}, 30 \mathrm{~nm}$, $20 \mathrm{~nm}$, and even $10 \mathrm{~nm}$, of which there was an overlap of particle size ranges according to the smallest and largest particles in a detailed and specific TEM image with a defined bar scale. The largest and smallest particles with the largest and smallest sizes were the limits of the nanoparticle system fabricated in EG. In our process, we prepared a homogeneous nanosystem of Au nanoparticles in a homogeneous mixture of EG and PVP [6,12]. Their common shapes were experimentally found to be the most typical spherical and polyhedral forms. The high roughness or/and flatness surfaces of the as-prepared spherical and polyhedral Au nanoparticles were clearly observed in their morphologies. Here, many Au nanoparticles possessed various large sizes and shapes, but were less than $100 \mathrm{~nm}$. In addition, the self-assembly, combination, and recrystallization of smaller Au nanoparticles less than $10 \mathrm{~nm}$ could lead to large nanoparticles, even greater than $100 \mathrm{~nm}$. The largest particles were around $50 \mathrm{~nm}$ by the self-assembly of smaller particles [25,26]. Therefore, the large particles result from the combinations of smaller particles, which would be through mechanical motions, collisions, combination, recrystallization, and formations.

In our particle size measurement, a strictly scientific study of particle size and a large number of $\mathrm{Au}$ nanoparticles fabricated by the designed polyol processes led to being able to obtain information for any significant improvement of further synthetic processes. To calculate the particle size of the as-prepared Au nanoparticles, it is very necessary to calculate the maximum and minimum sizes of the largest and smallest Au particles, a group of the Au particles seen, and all Au particles measured on that TEM image (Figure 2a). There are many ways to calculate the average particle sizes of large particles, medium size particles, and small particles (Figure $2 \mathrm{a}-\mathrm{d}$ ). Figure $2 \mathrm{~d}$ shows the rough calculation to arrange, in the order of size, the spherical particles, only a small amount of five Au particles from largest to smallest size, which were about 59.00, 55.52, 42.86, 31.50, and $11.69 \mathrm{~nm}$, respectively, which corresponded roughly to the three nanosized regions of particle sizes of Au nanoparticles based on the evidence of the original TEM image in the ranges of 60, 50,40 , and $20 \mathrm{~nm}$, even the possible smallest nanosized range of $10 \mathrm{~nm}(1-10 \mathrm{~nm})$ when TEM images of Au nanoparticles were carefully calculated and analyzed in this context (Figure 2a). The average size was calculated to be about $40 \mathrm{~nm}$, corresponding to five $\mathrm{Au}$ particles. It is certain that this is the simplest way to determine particle size. In addition, Figure $2 \mathrm{~d}$ shows mostly spherical shapes of Au nanoparticles with definite sizes. The critical issue indicated that the crystal formation of large crystal particles, medium crystal particles, and very small crystal particles was confirmed. The spherical and cubic particle shapes were the best forms to calculate the particle sizes here. The average grain size (d), standard deviation (std), and grain number (n) were calculated in Figure 3, respectively. In Figure 3a, we obtained the average size of about $28( \pm 27.65 \mathrm{~nm})$ when 58 particles of various sizes from small to large particles were used to calculate the histogram. We obtained an average size of about $56.90 \pm 11 \mathrm{~nm}$ after using about 82 particles of only large particles, which indicated that there were nanosize ranges $<60 \mathrm{~nm}$. Figures 4 and 5 present a special case of calculation where the average size was calculated to be about $30.20 \mathrm{~nm}$ $( \pm 9.43 \mathrm{~nm})$, which is relatively similar to the value $(\mathrm{d})$ calculated in Figure $3 \mathrm{a}$. 

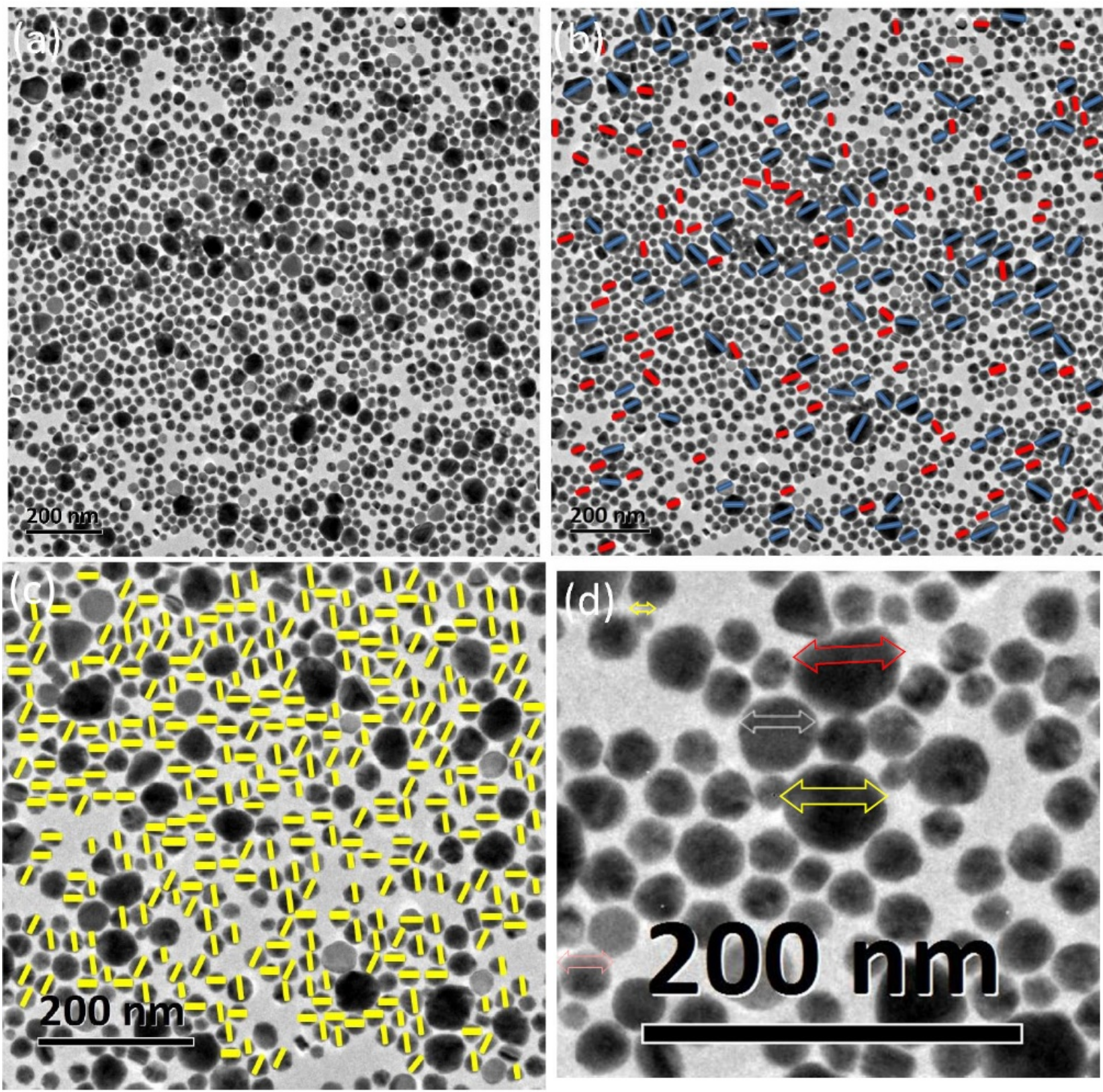

Figure 2. (a) TEM original image of the as-prepared Au nanoparticles. Scale bar: $200 \mathrm{~nm}$. (b,c) Selected nanoparticles of the original TEM image for the determination of the size and the shape (the use of five particles). (b) Small, medium, and large Au nanoparticles as well as the smallest and largest Au nanoparticles according to the colors of the scale indicators, respectively. (c) Medium Au nanoparticles. (d) Simplification of the determination of particle sizes of the as-prepared Au nanoparticles.

Figure 6 clearly indicates the typical HRTEM images of three Au nanoparticles showing their lattice fringes of about $0.248 \mathrm{~nm}$, which were assigned to the inter-fringe distance of the crystal planes in the FCC Au nanocrystal. The complexity of the Au atom arrangement on the crystal surface of one such Au nanoparticle is of high interest to scientists and researchers in the further investigation of TEM. In terms of size and surface investigation, it is clear that XRD determined the Au crystal structure of the prepared products, while HRTEM indicated the lattice fringes of Au nanoparticles. The self-assembly phenomenon of $\mathrm{Au}$ nanoparticles is popular in various nanosize ranges [25,26]. It is predicted that the large particles were formed by self-assembly via the motions, collisions, and combination of smaller particles. Therefore, the EG-type polyol in this case can be the best environment 
for Au nanoparticles synthesized in terms of the various sizes, various shapes, and the various morphologies. The experimental crystal formation of Au nanoparticles in the chemical reaction was confirmed by self-attachment, self-aggregation, and self-assembly of the much smaller nanoparticles [25-27]. The polyols or alcohols were considered as an excellent environment for the existence of the engineered particles such as the case of the Pd nanoparticles [28]. It is suggested that Au nanoparticles were generated in a homogeneous mixture of EG and PVP during chemical mixing and synthetic reaction. In potential clinical applications, the determination of particle size and crystal structure of $\mathrm{Au}$ nanoparticles has become increasingly significant as they are used in virus and COVID-19 pandemic research [29], for example, human ACE2-Au-virustrap-nanostructures for test kits in the capture of SARS-CoV-2 and single-virus SERS detection.
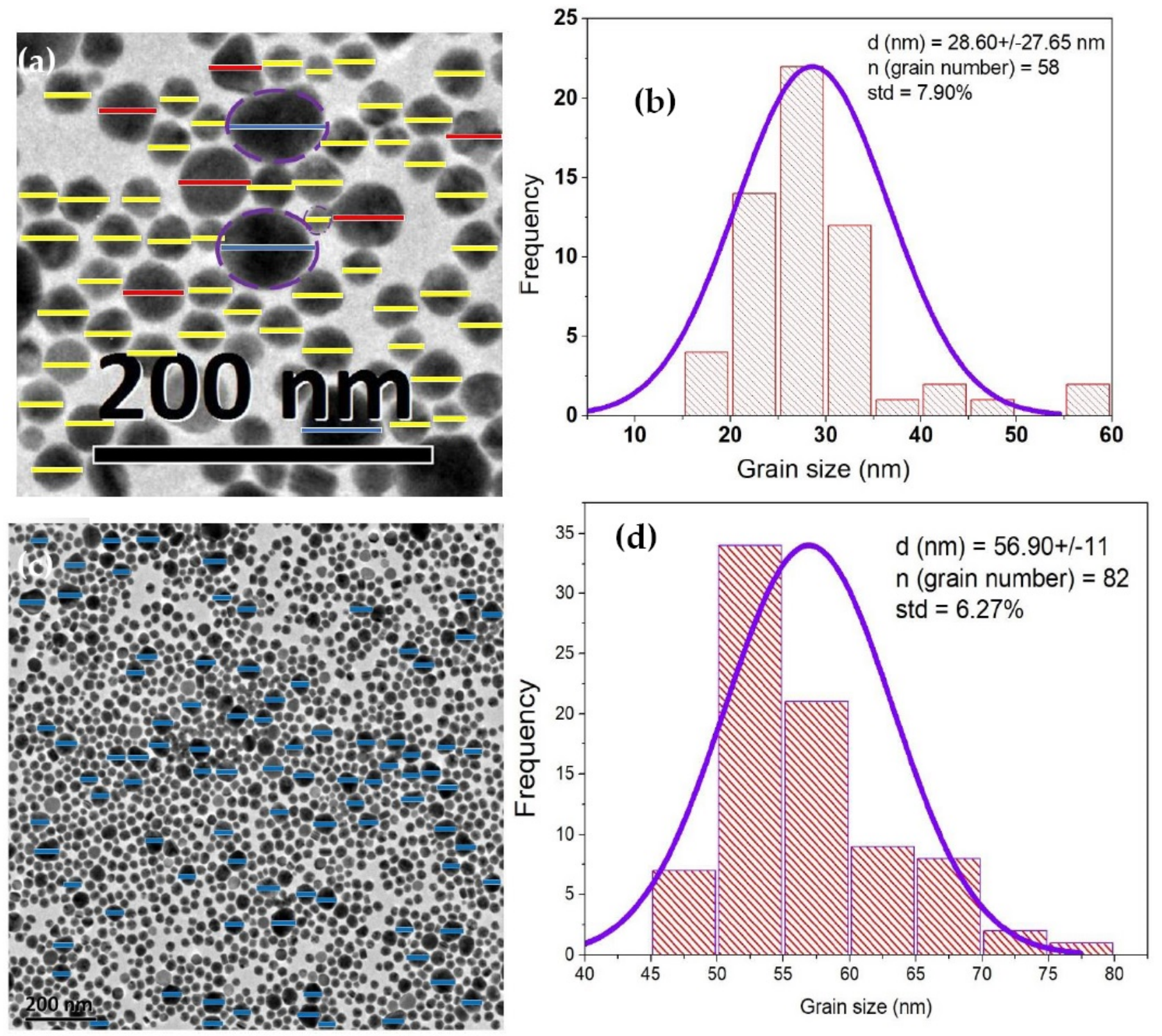

Figure 3. (a,b) Determination of particle size from the TEM image of the various kinds of as-prepared Au nanoparticles from the smallest to largest particles. The histogram of size particle distribution. Scale bar: $200 \mathrm{~nm}$. (c,d) Determination of particle size from the TEM image of the various kinds of as-prepared Au nanoparticles from the large particles (the use of 58 large particles). The histogram of the size particle distribution (using 82 particles). Scale bar: $200 \mathrm{~nm}$. 


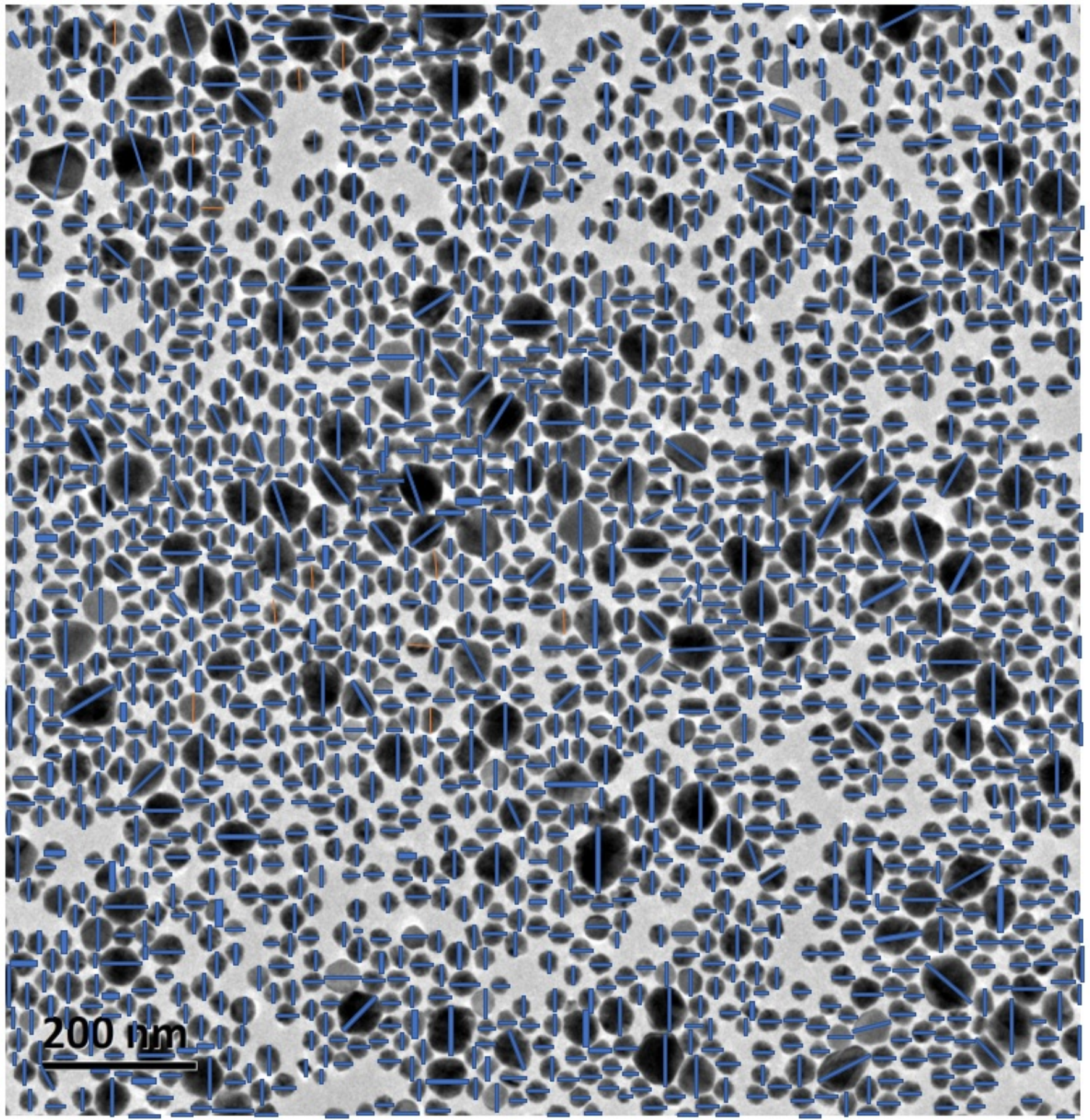

Figure 4. Particle size determination of all the particles that can be observed in the original TEM image (using a large number of Au particles, more than 1700 Au particles). Scale bar: $200 \mathrm{~nm}$. 


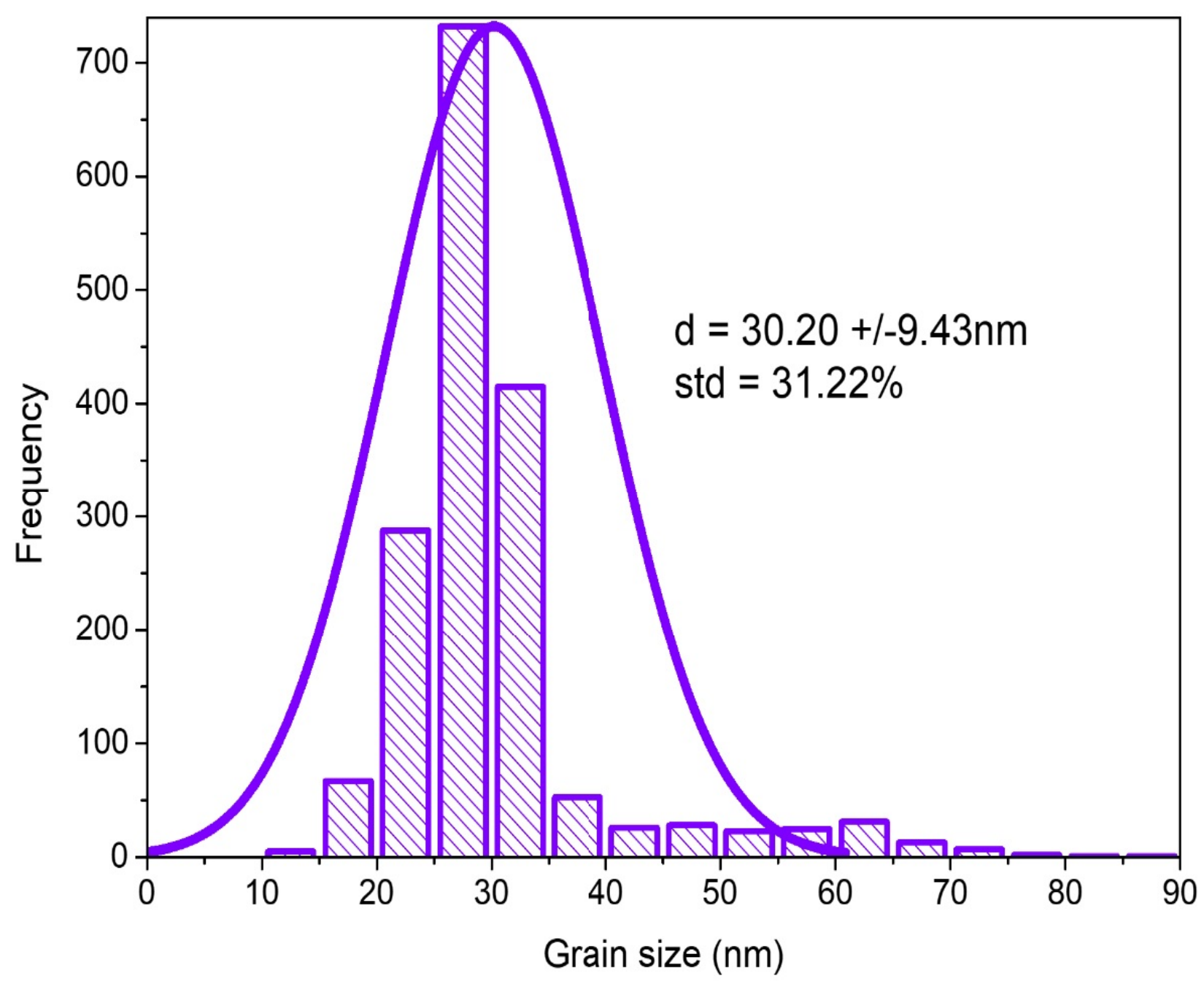

Figure 5. The histogram of size particle distribution (using all the particles, more than 1700 Au particles that could be observed in the TEM image in Figure 4) using a large number of Au particles.
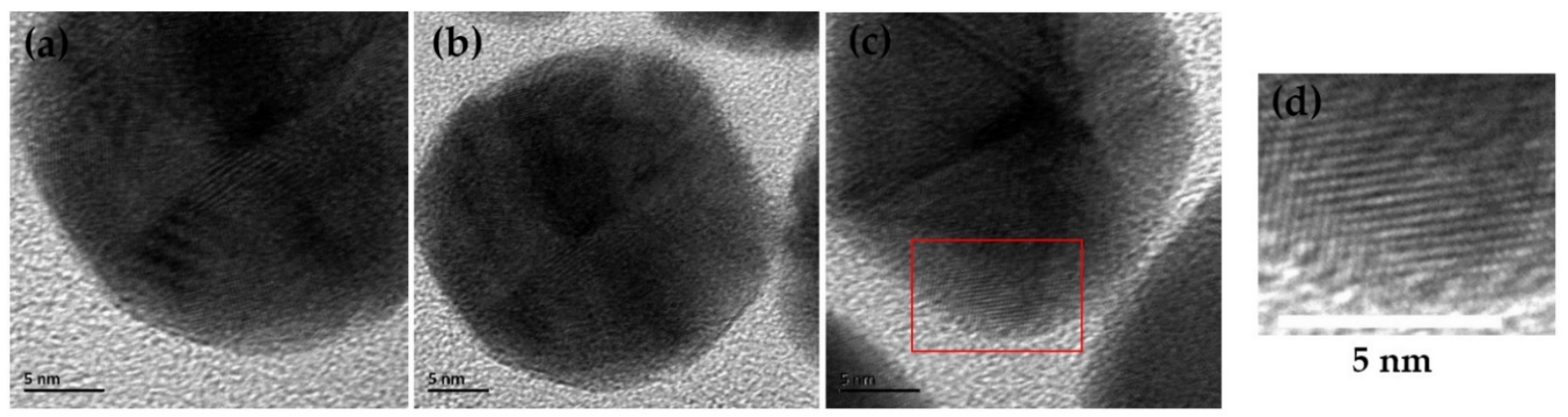

Figure 6. (a-c) High resolution TEM images of Au nanoparticles. (d) HRTEM image of a selected area of lattice fringes with a scale bar of $5 \mathrm{~nm}$. Gold atomic arrangement for a crystallographic plane is of very high interest in the Au crystal nanostructure at the atomic level.

\section{Conclusions}

In this research, we presented the formation of Au nanoparticles synthesized in EG and PVP with the addition of $\mathrm{NaBH}_{4}$. The Au nanoparticles ranged in size up to $60 \mathrm{~nm}$, with an average size of about $30 \mathrm{~nm}$. In the process of manually calculating each particle, errors can 
occur despite the careful particle-size calculation of the experimenter. However, there were various kinds of Au nanoparticles shaped in the various ranges of $50 \mathrm{~nm}, 40 \mathrm{~nm}, 30 \mathrm{~nm}$, and even $10 \mathrm{~nm}$. Typical shapes for nanosized particles in this range were spherical and polyhedral. It is evident that the modified polyol methods can be promisingly used with the addition of a small content of structure-controlling (i.e., $\mathrm{NaBH}_{4}$ ) and other chemical reagents in any synthetic process. The spherical and cubic shapes were suggested to be the best forms of particle size calculation in this study. In this research, it is suggested that the TEM method is very important to calculate the parameters of particle size, number of $\mathrm{Au}$ nanoparticles, and shape of the Au nanoparticles. Therefore, this research also suggests that the experimenter must choose the experimental conditions of $\mathrm{pH}$, fixed reaction volumes of precursors in solvent (for the effective formation of nanoparticles), the solvent (EG, PEG, or other polyols for media generating nanoparticles), surfactant (PVP, CTAB, other protective agents for nanoparticles), reaction time (fast, moderate, or short time), and stirring speed for synthesis.

Author Contributions: Experimenting, collecting data and results, writing the whole original manuscript, discussion: N.V.L.; Checking and discussing the research results: N.T.N.H., Y.Y., M.N., N.Q.T.N., L.H.P., N.H.T. and H.V.C.; Funding, Y.Y. (China, SICCAS) and N.T.N.H. (Vietnam, TDMU) All authors have read and agreed to the published version of the manuscript.

Funding: This research was funded by Sai Gon University, grant number TĐ2020-19.

Institutional Review Board Statement: Not applicable.

Informed Consent Statement: Not applicable.

Data Availability Statement: Not applicable.

Acknowledgments: Our research was supported by Saigon University through grant number TĐ2020-19. The author (N.V.L.) appreciates the kind research assistance from the Universities and Institutes of Japan (Nagoya Institute of Technology, NITECH; Kyushu University, Kyoto University), China (Shanghai Institute of Ceramics, Chines Academy of Sciences), and Vietnam (Sai Gon University, Thu Dau Mot University, Ho Chi Minh City Institute of Physics, National Foundation for Science \& Technology Development) for their financial support during his research of the controlled synthesis of precious nanoparticles by modified polyol methods. Our research was supported by the National Foundation for Science and Technology Development of Vietnam (NAFOSTED). N.V.L. is very grateful to P.M. Tien as the Director of HCMC IOP, and C.H. Thien as the scientific chairman for their very kind support when he carried out his experimental work at HCMC IOP. The authors would like to thank Jeremy Evert at Southwestern Oklahoma State University, USA, for his diligent proofreading of this article.

Conflicts of Interest: The authors declare no conflict of interest.

\section{References}

1. Burda, C.; Chen, X.; Narayanan, R.; El-Sayed, M.A. Chemistry and Properties of Nanocrystals of Different Shapes. Chem. Rev. 2005, 105, 1025-1102. [CrossRef] [PubMed]

2. Fiévet, F.; Ammar-Merah, S.; Brayner, R.; Chau, F.; Giraud, M.; Mammeri, F.; Peron, J.; Piquemal, J.-Y.; Sicard, L.; Viau, G. The polyol process: A unique method for easy access to metal nanoparticles with tailored sizes, shapes and compositions. Chem. Soc. Rev. 2018, 47, 5187-5233. [CrossRef]

3. Ammar, S.; Fiévet, F. Polyol Synthesis: A Versatile Wet-Chemistry Route for the Design and Production of Functional Inorganic Nanoparticles. Nanomaterials 2020, 10, 1217. [CrossRef] [PubMed]

4. Kelly, K.L.; Coronado, E.; Zhao, L.L.; Schatz, G.C. The optical properties of metal nanoparticles: The influence of size, shape, and dielectric environment. J. Phys. Chem. B 2003, 107, 668-677. [CrossRef]

5. Long, N.V.; Ohtaki, M.; Yuasa, M.; Yoshida, S.; Kuragaki, T.; Thi, C.M.; Nogami, M. Synthesis and Self-Assembly of Gold Nanoparticles by Chemically Modified Polyol Methods under Experimental Control. J. Nanomater. 2013, $2013,793125$.

6. Long, N.V.; Yong, Y.; Lin, Z.L.; Cao, Y.; Thi, C.M.; Nogami, M. Controlled Synthesis of Gold Nanoparticles by Modified Polyol Methods for Plasmonic Applications. In New Developments in Gold Nanomaterials Research; Xu, Z., Yong, J.K.C., Eds.; Series: Nanotechnology Science and Technology; Nova Science Publishers, Inc.: Hauppauge, NY, USA, 2016; Chapter 2; pp. $25-37$. 
7. Long, N.V.; Yong, Y.; Lin, Z.L.; Cao, Y.; Thi, C.M.; Nogami, M. New Developments of Gold Nanoparticles in Plasmonic Sensing. In New Developments in Gold Nanomaterials Research; Xu, Z., Yong, J.K.C., Eds.; Series: Nanotechnology Science and Technology; Nova Science Publishers, Inc.: Hauppauge, NY, USA, 2016; Chapter 7; pp. 115-146.

8. Long, N.V.; Thi, C.M.; Nogami, M. The recent patents and highlights of functionally engineered nanoparticles for potential applications in biology, medicine, and nanomedicine. Curr. Phys. Chem 2014, 4, 173-194. [CrossRef]

9. Long, N.V.; Chien, N.D.; Hayakawa, T.; Hirata, H.; Lakshminarayana, G.; Nogami, M. The Synthesis and Characterization of Platinum Nanoparticles: A Method of Controlling the Size and Morphology. Nanotechnology 2009, 21, 35605. [CrossRef] [PubMed]

10. Huang, C.; Zhao, H.; Peng, Z.; Zheng, B.; Zhang, C.; Wang, J. One-Step Fabrication of Highly Dense Gold Nanoparticles on Polyamide for Surface-Enhanced Raman Scattering. Appl. Surf. Sci. 2021, 561, 149856. [CrossRef]

11. Rodrigues, T.S.; Zhao, M.; Yang, T.H.; Gilroy, K.D.; da Silva, A.G.; Camargo, P.H.; Xia, Y. Synthesis of colloidal metal nanocrystals: A comprehensive review on the reductants. Chem. Eur. J. 2018, 24, 16944-16963. [CrossRef] [PubMed]

12. Yue, H.; Zhao, Y.; Ma, X.; Gong, J. Ethylene glycol: Properties, synthesis, and applications. Chem. Soc. Rev. 2012, 41, 4218-4244. [CrossRef]

13. Wei, M.-Z.; Deng, T.-S.; Zhang, Q.; Cheng, Z.; Li, S. Seed-Mediated Synthesis of Gold Nanorods at Low Concentrations of CTAB. ACS Omega 2021, 6, 9188-9195. [CrossRef] [PubMed]

14. Daniel, M.-C.; Astruc, D. Gold Nanoparticles: Assembly, Supramolecular Chemistry, Quantum-Size-Related Properties, and Applications toward Biology, Catalysis, and Nanotechnology. Chem. Rev. 2004, 104, 293-346. [CrossRef] [PubMed]

15. Suchomel, P.; Kvitek, L.; Prucek, R.; Panacek, A.; Halder, A.; Vajda, S.; Zboril, R. Simple Size-Controlled Synthesis of Au Nanoparticles and Their Size-Dependent Catalytic Activity. Sci. Rep. 2018, 8, 4589. [CrossRef] [PubMed]

16. Lin, S.; Lin, X.; Shang, Y.; Han, S.; Hasi, W.; Wang, L. Self-Assembly of Faceted Gold Nanocrystals for Surface-Enhanced Raman Scattering Application. J. Phys. Chem. C 2019, 123, 24714-24722. [CrossRef]

17. Nwahara, N.; Achadu, O.J.; Nyokong, T. In-Situ Synthesis of Gold Nanoparticles on Graphene Quantum Dots-Phthalocyanine Nanoplatforms: First Description of the Photophysical and Surface Enhanced Raman Scattering Behaviour. J. Photochem. Photobiol. A Chem. 2018, 359, 131-144. [CrossRef]

18. Bai, L.; Ouyang, Y.; Song, J.; Xu, Z.; Liu, W.; Hu, J.; Wang, Y.; Yuan, F. Synthesis of Metallic Nanocrystals: From Noble Metals to Base Metals. Materials 2019, 12, 1497. [CrossRef]

19. González-Rubio, G.; Hilbert, H.; Rosenberg, R.; Ni, B.; Fuhrer, L.; Cölfen, H. Simple Determination of Gold Nanocrystal Dimensions by Analytical Ultracentrifugation via Surface Ligand-Solvent Density Matching. Nanomaterials 2021, $11,1427$. [CrossRef]

20. Hossain, M.K. Nanoassembly of Gold Nanoparticles: An Active Substrate for Size-Dependent Surface-Enhanced Raman Scattering. Spectrochim. Acta Part A Mol. Biomol. Spectrosc. 2020, 242, 118759. [CrossRef]

21. Long, N.V.; Thi, C.M.; Nogami, M.; Ohtaki, M. Novel issues of morphology, size, and structure of Pt nanoparticles in chemical engineering: Surface attachment, aggregation or agglomeration, assembly, and structural changes. New. J. Chem. 2012, 36, 1320-1334. [CrossRef]

22. Long, N.V.; Chien, N.D.; Uchida, M.; Matsubara, T.; Randy, J.; Masayuki, N. Directed and random self-assembly of Pt-Au nanoparticles. Mater. Chem. Phys. 2010, 124, 1193-1197. [CrossRef]

23. Long, N.V.; Ohtaki, M.; Uchida, M.; Jalem, R.; Hirata, H.; Chien, N.D.; Nogami, M. Synthesis and characterization of polyhedral Pt nanoparticles: Their catalytic property, surface attachment, self-aggregation and assembly. J. Colloid Interface Sci. 2011, 359, 339-350. [CrossRef] [PubMed]

24. Favier, I.; Pla, D.; Gómez, M. Palladium nanoparticles in polyols: Synthesis, catalytic couplings, and hydrogenations. Chem. Rev. 2019, 120, 1146-1183. [CrossRef] [PubMed]

25. Yang, Y.; Peng, Y.; Lin, C.; Long, L.; Hu, J.; He, J.; Zeng, H.; Huang, Z.; Li, Z.Y.; Tanemura, M.; et al. Human ACE2-Functionalized Gold "Virus-Trap" Nanostructures for Accurate Capture of SARS-CoV-2 and Single-Virus SERS Detection. Nano-Micro Lett. 2021, 13, 109. [CrossRef] [PubMed]

26. Crystallography Open Database. Available online: http:/ / www.crystallography.net/cod/9008463.html (accessed on 25 September 2021).

27. VESTA. Available online: https://jp-minerals.org/vesta/en/ (accessed on 25 September 2021).

28. Momma, K.; Izumi, F. VESTA 3 for three-dimensional visualization of crystal, volumetric and morphology data. J. Appl. Cryst. 2011, 44, 1272-1276. [CrossRef]

29. Gharbi, K.; Mezni, A.; Collière, V.; Philippot, K.; Amiens, C. Controlled Synthesis of Anisotropic Gold Nanoparticles by a Simple Polyol Process and Their Related Optical Properties. J. Tunis. Chem. Soc. 2017, 4, 335-342. 